\title{
Status of Development of the Insertion Devices for ELETTRA
}

\author{
C.Poloni, R.Bracco, B.Diviacco, R.P.Walker and D.Zangrando \\ Sincrotrone Trieste, Padriciano 99, 34012 Trieste, Italy
}

\section{Abstract}

The status of development of insertion devices for the 1.5-2 $\mathrm{GeV}$ synchrotron radiation source ELETTRA is described, including details of the prototype undulator, multipole wiggler, mechanical support structure and vacuum chamber. Plans for a novel source of circularly polarized radiation are also presented.

\section{INTRODUCTION}

A $1.5-2 \mathrm{GeV}$ third generation synchrotron radiation source, ELETTRA, is under construction in Trieste, Italy $[1,2]$. The storage ring design has been optimized for the inclusion of up to 11 insertion devices (IDs), which will provide high brightness radiation from pure permanent magnet undulators (U), hybrid multipolc wigglcrs (W), and special sources of circularly polarized radiation. Table 1 summarizes the main parameters of the devices presently forseen for the initial phase of operation.

Table 1. Preliminary ELETTRA insertion device parameters.

Beamline Type-Period $(\mathrm{cm})$ Field $(\mathrm{T}) \quad$ No. of Periods

$\begin{array}{llll}\text { Superesca } & \text { U 5.6 } & 0.60 & 81 \\ \text { Surface diffraction } & \text { U 7.3 } & 0.77 & 63 \\ \text { Spectromicroscopy } & \text { U 7.3 } & 0.77 & 63 \\ \text { Photoemission } & \text { U 12.5 } & 0.49 & 36 \\ \text { Diffraction } & \text { W 14.0 } & 1.55 & 30\end{array}$

\section{INSERTION DEVICE SUPPORT STRUCTURE}

For reasons of ease of construction, flexibility in use and cost, a standard mechanical support structure for both undulators and wigglers will be used $[3,4]$. The structure is $1.5 \mathrm{~m}$ long, so that each of the 11 ID straight sections in ELETTRA will accommodate up to 3 such structurcs. Following a design study made by Sincrotrone Trieste [4], a prototype carriage was constructed by CONTEK, Italy, and delivered in June 1990 (see fig. 1). Since then, a series of detailed measurements has been carried out to assess the mechanical performance, including the effect of a magnetic load, as well as further finite element structural calculations. The mechanical measurements were made using a HP 5528A laser interferometer and two Heidenhain optical rulers. The following values, that are within the specification, have becn measured independent of magnetic load : gap setting accuracy $\pm 10 \mu \mathrm{m}$, gap reproducibility $\therefore 10 \mu \mathrm{m}$, flatness of the reference surfaces 30 $\mu \mathrm{m}$, parallelism of the I-beams $60 \mu \mathrm{m}$ (for gaps between the Ibcams $\leq 550 \mathrm{~mm}$ ).

The measured deformation of the structure due to the magnetic load of the undulator prototype has been found to be significantly larger ( 5 times) than the value foreseen with the first structural model used during the design stage (using beam elements) [4] but in good agreement ( $7 \%$ of difference including the load determination error) with a more detailed finite element model prepared to take into account the true 3D geometry of the structure [5]. A gap variation along the beam axis of $1.4 \mu \mathrm{m} / \mathrm{kN}$ and a parallelism error of $12.7 \mu \mathrm{rad} / \mathrm{kN}$ in the plane normal to the beam axis are induced by the magnetic force. This leads to a total gap error of $\pm 5.5 \mu \mathrm{m}$ in the case of the undulator prototype which is higher than the specified value but is still acceptable. Calculations made with the accurate structural model have shown that a vertical thermal gradient of $1{ }^{\circ} \mathrm{C} / \mathrm{m}$ and an ambient temperature stability of \pm $2.5^{\circ} \mathrm{C}$ do not cause unacceptable deformations of the insertion device support structure.

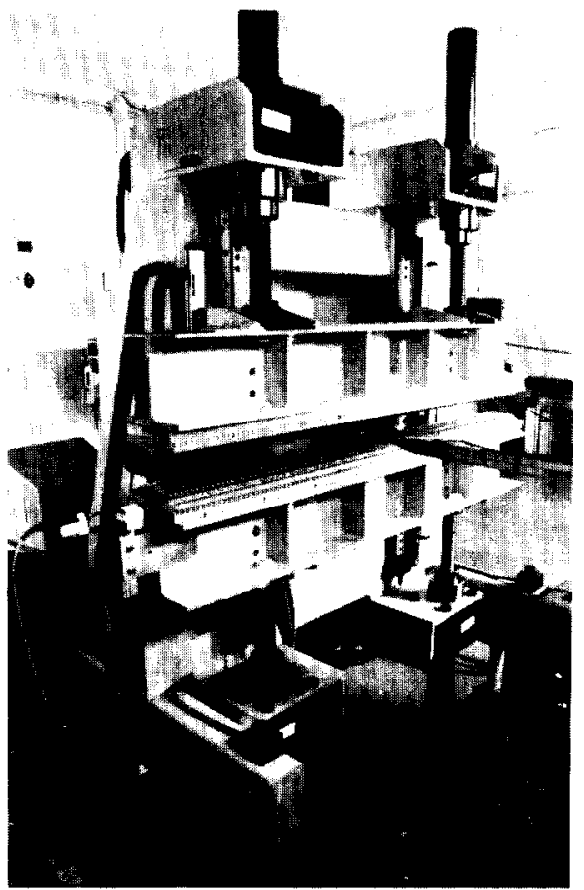

Figure 1. Prototype of ELETTRA insertion device support structure and undulator magnet.

\section{UNDULATOR PROTOTYPE}

Initial measurements of the $\mathrm{NdFcB}$ blocks for a $56 \mathrm{~mm}$ period pure permanent magnet undulator, using a Helmholz coil system to determine the total block magnetizations and a Hall plate system to make point measurements, were described previously [6]. It was clear from the results of these and later measurements that to the level of accuracy required the blocks could not be modelled as ideal CSFM material, even if different magnetization values were used for the upper and lower faces of the blocks. Instead a purely empirical approach had to be adopted, but still relying on the fact that to a good approximation the field from different blocks superimposes 
linearly. In order to allow the main field component in the undulator to be calculated at the peaks and zeros each block has been measured at 21 points above and below the block, with a spacing of one quarter of the period along the beam direction, at a distance corresponding to the minimum magnetic gap (20 $\mathrm{mm}$ ). In addition a new stretched-wire flipping coil bench has been used to measure the field integrals in both planes, above (u) and below (d) the blocks, along the beam direction and displaced transversely by $\pm 15 \mathrm{~mm}$. The average time required per block was $14 \mathrm{~min}$. for the Hall plate and $30 \mathrm{~min}$. for the flipping coil. Table 2 shows the results from the flipping coil for " $\mathrm{A}$ " and " $\mathrm{C}$ " blocks (vertical magnetization) and for "B" blocks (horizontal magnetization) [6].

Table 2. Results of measurements of the ficld integrals for the prototype undulator blocks, in units of $\mu^{\prime} \mathrm{Tm}$.

\begin{tabular}{|c|c|c|c|c|c|c|}
\hline & \multicolumn{2}{|c|}{ A (111 blocks) } & \multicolumn{2}{|c|}{$\mathrm{B}$ (117 blocks) } & \multicolumn{2}{|c|}{ C (24 blocks) } \\
\hline & mean & $\mathrm{rms}$ & mean & $\mathrm{rms}$ & mean & $\mathrm{rms}$ \\
\hline $\mathrm{Ix}^{\mathrm{u}}-\mathrm{Ix}^{\mathrm{d}}$ & 2.0 & 18.1 & -39.2 & 60.4 & -2.3 & 10 \\
\hline$\left(I x^{\mathrm{u}}+\mathrm{Ix}^{\mathrm{d}}\right) / 2$ & -2.2 & 68.5 & -8.7 & 71.4 & -1.3 & 16. \\
\hline $\mathrm{Iy}^{\mathrm{u}}-\mathrm{I} \mathrm{y}^{\mathrm{d}}$ & 17.1 & 236.9 & 0.7 & 216.4 & -1.5 & 151. \\
\hline$\left(1 y^{\mathrm{u}}+\mathrm{Iy}^{\mathrm{d}}\right) / 2$ & 2956.1 & 28.5 & 0.3 & 51.1 & 1474.1 & 8.3 \\
\hline
\end{tabular}

A computer program has been written to arrange the blocks in the structure using a simulated annealing algorithm [7] involving the following components to the "cost function" that was minimized: (a) ms field error at the peaks $(\Delta \mathrm{B} / \mathrm{B})$ and crossing-points, (b) first and second field integrals, (c) horizontal trajectory 'straightness' and (d) rms vertical displacement. A total cpu time of 5 hours was required for calculating the arrangement of the 222 blocks in the $1.5 \mathrm{~m}$ undulator.

The undulator was constructed according to the specified arrangement and then measured using both benches. Figure 2 shows the measured field and trajectory at minimum gap, and Table 3 summarizes various parameters as a function of magnet gap.
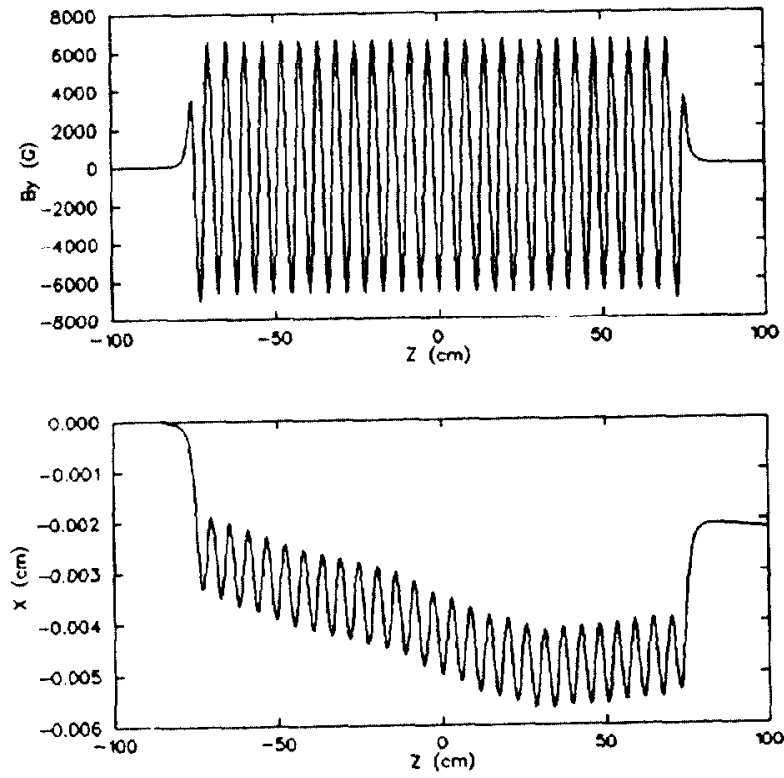

Figure 2. Measured magnetic field (upper) and trajectory (lower) in the prototype undulator at minimum gap ( $2 \mathrm{GeV})$.
Compared to a random configuration of the blocks the performance is very much better, at least an order of magnitude for $\triangle B / B$, but not as good as predicted, particularly for the field integrals. $A$ factor which probably contributed to this error is the variation in temperature during the block measurements $\left( \pm 4.5^{\circ} \mathrm{C}\right)$. Attempts to measure and correct for the temperature variation have been unsuccessful, possibly due to the thermal time constant of the blocks, about 20 minutes, which means that the surface temperature can be different from the bulk. Use of a temperature stabilized laboratory in the future should eliminate this error.

Table 3. Results of initial measurements of the ELETTRA prototype undulator.

$\begin{array}{lcccc}\text { gap }(\mathrm{mm}) & \mathrm{Bo}(\mathrm{T}) & \Delta \mathrm{B} / \mathrm{B} \mathrm{rms} \% & \mathrm{Ix}(\mu \mathrm{T} \mathrm{m}) & \text { Iy }(\mu \mathrm{T} \mathrm{m}) \\ 20 & 0.66 & 0.34 & 398 . & 70.5 \\ 30 & 0.37 & 0.41 & 306 . & 35 . \\ 50 & 0.12 & 0.84 & 178 . & -15.6\end{array}$

To improve the quality of the undulator field experiments are presently being carried out using shims made from $\mathrm{Fe}-\mathrm{Si}$ laminations, placed on the surface of the blocks. So far, very good agreement has been obtained between measured changes in both field amplitude and field integral and model calculations [8]. Efforts are presently being directed towards compensation of residual errors over a range of operating gap. Correction coils for fine adjustment of field integrals have also been fabricated and will soon be tested.

\section{MULTIPOLE WIGGLER PROTOTYPE}

The optimization of the wiggler parameters to give the maximum field level with the given constraints on gap and radiation opening angle resulted in a period of $125 \mathrm{~mm}$, with a predicted field of $1.48 \mathrm{~T}$ [3]. Since then the plan to include an additional experimental station on the wiggler beamline which will take radiation from the outer part of the radiation fan has necessitated a modification of the parameters, to take into account the effect of the third harmonic field component on the critical energy of the radiation emitted off-axis [9]. Figure 3 shows the relation between field and angle, calculated using POISSON, for the original design which has a third harmonic component (B3/B0) of $12 \%$.

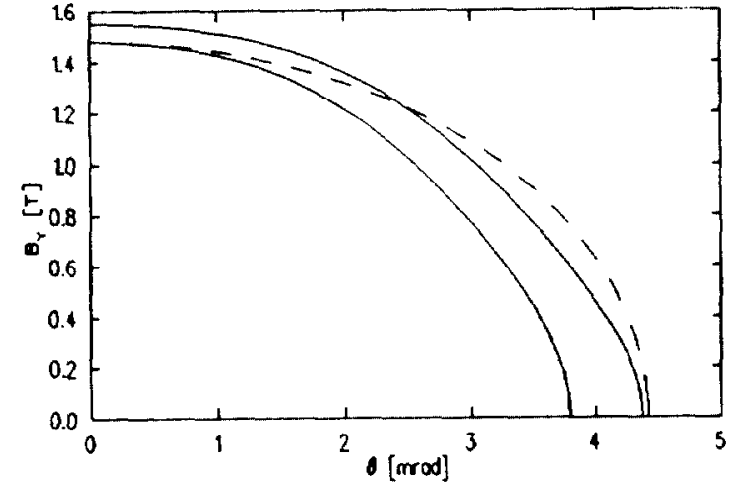

Figure 3. Field amplitude as a function of horizontal emission angle for the previous multipole wiggler design (lower solid curve), a sinusoidal model with the same field amplitude (dotted) and the present design (upper solid curve). 
It can be seen that the field (and hence critical energy) is significantly reduced for angles above about $2.5 \mathrm{mrad}$, and that the total radiation angle is reduced by a factor 0.87 , compared to the case of a purely sinusoidal field distribution. To overcome these effects the period length has been increased to $140 \mathrm{~mm}$ and the field to $1.55 \mathrm{~T}$, keeping the same transverse block dimensions. Figure 4 shows that the modified design gives significantly improved performance at off-axis angles. The increase in field compensates the reduction in number of full poles from 21 to 19 per section at energies above $10 \mathrm{keV}$. Components to construct a $0.5 \mathrm{~m}$ prototype will be delivered by the end of May, to test design calculations, assembly procedures, and different end configurations.

\section{CIRCULARLY POLARIZED RADIATION SOURCE}

Various schemes have been studied for the production of circularly polarized radiation $[3,10]$, in particular those that satisfy users' demands for a relatively broad spectral range (200 $\mathrm{eV}-1 \mathrm{keV})$ with a rapid variation of the helicity $(>20 \mathrm{~Hz})$. The solution favoured at present is a novel electromagnetic clliptical wiggler (see fig. 4), similar to the elliptical wiggler of ref. [11], except that the horizontal field component is generated by an a.c. electromagnet in order to modulate the helicity of the on-axis radiation.

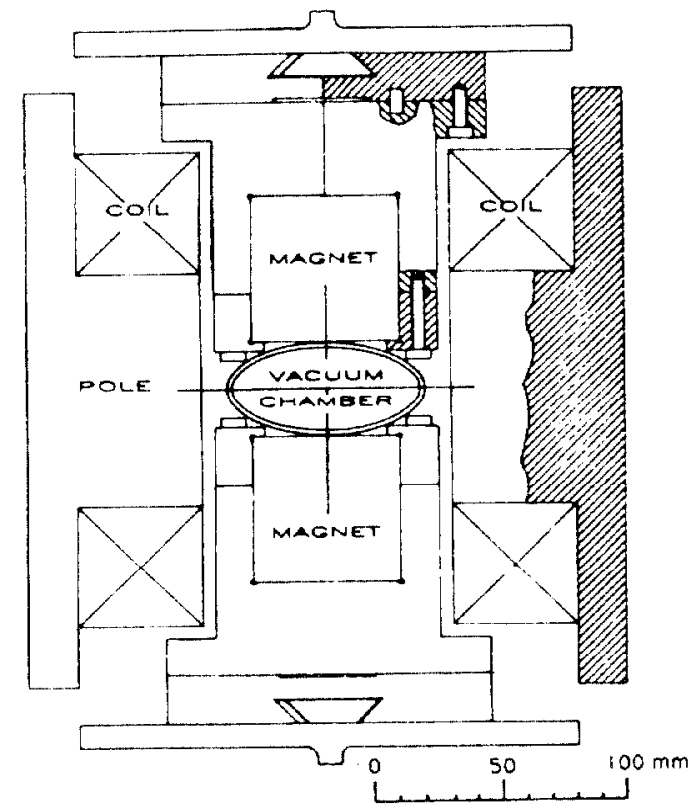

Figure 4. Preliminary design of an electromagnetic elliptical wiggler source of circularly polarized radiation.

The horizontal gap is necessarily large in order to allow space for the pure permanent magnet structure which produces the vertical wiggler field, and so a relatively large period has been chosen $(230 \mathrm{~mm})$ to reduce power supply demands. With this period a maximum field of $0.6 \mathrm{~T}$, in order to restrict the opening angle of the radiation, gives an acceptable critical energy of up to $1.6 \mathrm{keV}(2 \mathrm{GeV})$. Table 3 gives preliminary parameters for the device, which using a special ID support structure $3 \mathrm{~m}$ long will give 25 times the flux of a bending magnet source with a modulation rate up to $100 \mathrm{~Hz}$. POISSON calculations have been carried out to calculate ficld amplitude and inductance. A prototype will be constructed in the following months using existing permanent magnet blocks and an a.c. power supply.

Table 3. Preliminary parameters for an electromagnetic elliptical wiggler, at $100 \mathrm{~Hz}$ excitation.

$\begin{array}{lll}\text { Period } & 0.23 & \mathrm{~m} \\ \text { Number of full poles } & 25 & \\ \mathrm{Bx}, \mathrm{By} & 0.0466,0.61 & \mathrm{~T} \\ \mathrm{Kx}, \mathrm{Ky} & 1.0,13.2 & \\ \text { Inductance, total } & 2.5 & \mathrm{mH} \\ \text { Ampere-turns per pole, peak } & 5000 & \mathrm{~A} \\ \text { Current, peak } & 250 & \mathrm{~A} \\ \text { Voltage, peak } & 432 & \mathrm{~V}\end{array}$

The effect of the device on the dynamic aperture of the storage ring has been calculated and is tolerable [12].

\section{INSERTION DEVICE VACUUM CHAMBER}

Following the design and specification of a narrow gap (15 $\mathrm{mm}$ internal, $20 \mathrm{~mm}$ external) vacuum chamber employing NEG pumping $[4,13]$, an order for the construction of a $2.4 \mathrm{~m}$ half-length prototype vacuum chamber has been placed with E.ZANON, Italy, including a tapered section $240 \mathrm{~mm}$ long which incorporates a pumping tee. The chamber will be constructed from stainless steel 316 LN. The elliptical beam chamber will be obtained by deformation of a $54 \mathrm{~mm}$ diameter circular pipe, formed from $1.5 \mathrm{~mm}$ thick sheet material, with machined pumping slots $16 \mathrm{~mm}$ long $\times 10 \mathrm{~mm}$ high spaced every $40 \mathrm{~mm}$. The ante-chamber will be formed from $3.5 \mathrm{~mm}$ sheet, machined then TIG welded to the beam chamber. After delivery in May 1991 and subsequent installation of the NEG pumping strips it will then undergo a thorough series of tests in the Vacuum Laboratory.

\section{REFERENCES}

[1] A.Wrulich, this Conference

[2] ELETTRA Conceptual Design Report, April 1989, Sincrotrone Trieste

[3] B.Diviacco and R.P.Walker, Proc. 2nd European Particle Accelerator Conference, Nice, June 1990, p. 1359

[4] C.Poloni and R.P.Walker, ibid, p. 1362

[5] C.Poloni, Report in preparation

[6] D.Zangrando and R.P.Walker, ibid, p. 1365

[7] A.D.Cox and B.P.Youngman, Proc. SPIE 582, p. 91

[8] J.Chavanne et. al., European Synchrotron Radiation Facility Report ESRF-SR/ID-89-32, Nov. 1989.

[9] W.Hassenzahl, private communication

[10] B.Diviacco and R.P.Walker, Nucl. Instr. Meth. Phys. Res. A292 (1990) 517.

[11] S.Yamamoto and H.Kitamura, Jpn. J. Appl. Phys. 26 (1987) L1613.

[12] L.Tosi and R.Nagaoka, this conference

[13] T.Monaci and C.Poloni, Sincrotrone Trieste Report ST/M-TN-90/18, Sep. 1990 